\title{
Non-pharmaco, non-invasive management of coronary no-reflow phenomenon
}

\author{
Santosh Kumar Sinha ${ }^{1}$, Mukesh Jitendra Jha ${ }^{2}$, Puneet Aggarwal ${ }^{3}$, Umeshwar Pandey², \\ Awadesh Kumar Sharma², Mahmodullah Razi ${ }^{2}$, Dibbendhu Khanra², Ramesh Thakur², Vinay Krishna²
}

${ }^{1}$ Department of Cardiology, LPS Institute of Cardiology, Kanpur, India ${ }^{2}$ Department of Cardiology, Sri Aurobindo Institute of Cardiology, Indore, India ${ }^{3}$ Department of Cardiology, RML Institute of Medical Science, New Delhi, India

Submitted: 10 July 2020

Accepted: 30 August 2020

Arch Med Sci Atheroscler Dis 2020; 5: e271-e278

DOI: https://doi.org/10.5114/amsad.2020.102424

Copyright @ 2020 Termedia \& Banach

\begin{abstract}
Introduction: No-reflow is an infrequent but dreaded complication of percutaneous coronary intervention $(\mathrm{PCl})$, where the culprit is obstruction of the downstream microvascular bed. The aim of this study was to evaluate the efficacy and safety of forceful injection of blood (autologous blood transfusion - $A B T$ ) in reversing no-reflow during $\mathrm{PCl}$ because data regarding its effectiveness is not available.
\end{abstract}

Material and methods: $100-120 \mathrm{ml}$ of blood was withdrawn through guiding catheter over 3 to 5 min using a $10 \mathrm{ml}$ syringe and re-infused by forceful injection over 3 min through it, and its efficacy was assessed at 10 min using TIMI flow grade and quantitative corrected TIMI frame count.

Results: In total 93 patients received ABT following no-reflow. Their clinical presentation was ST-elevation myocardial infarction (STEMI) $(n=61$; $65.6 \%)$, non-ST-elevation myocardial infarction (NSTEMI) ( $n=23 ; 24.7 \%$ ), and unstable angina $(n=9 ; 9.6 \%)$. It was observed among patients undergoing primary $\mathrm{PCl}(n=18 ; 19.3 \%)$, pharmaco-invasive $\mathrm{PCI}(n=27 ; 29 \%)$, rescue $\mathrm{PCl}(n=11 ; 11.8 \%)$, and $\mathrm{PCl}$ for cardiogenic shock $(n=5 ; 5.3 \%)$. A mean volume of $108 \pm 4 \mathrm{ml}$ blood was transfused. Commonest culprit vessel was left anterior descending artery $(n=51 ; 54.8 \%)$ followed by right coronary ( $n=29 ; 31.2 \%)$, left circumflex $(n=19 ; 10.8 \%)$, and saphenous vein grafts ( $n=3 ; 3.2 \%)$. Following ABT, TIMI 3 flow was successfully restored in $77(82.7 \%)$ patients. TIMI flow grade improved from 1.02 to 2.52 and CTIMI frame count decreased from $60.6 \pm 12$ to $16.1 \pm 6(p<0.001)$. ABT was well tolerated except transient hypotension $(n=17 ; 18.3 \%)$. Overall mortality was reported in 10 (10.7\%) patients at 1 year.

Conclusions: In this largest and only study to date, $A B T$ is a safe and highly effective approach to reverse no-reflow by raising driving pressure across the capillary bed.

Key words: percutaneous coronary intervention, acute coronary syndromes, TIMI frame count, TIMI flow, autologous blood transfusion, no-reflow.

\section{Introduction}

Restoration of patency of the culprit artery (infarct related artery) and re-flow of blood in the vessel occluded by thrombus by either pharmacological means using thrombolytics or mechanical means using percutaneous coronary intervention ( $\mathrm{PCl})$ is the gold standard of treatment in acute coronary syndrome $[1,2]$. Primary PCI successfully restores TIMI 3

\author{
Corresponding author: \\ Santosh Kumar Sinha FACC, \\ FESC, FSCAI \\ Department of Cardiology \\ LPS Institute of Cardiology \\ GSVM Med Coll, Kanpur, \\ India \\ Phone: 91-9670220088 \\ E-mail: fionasan@rediffmail. \\ com
}


flow (thrombolysis in myocardial infarction) in over $90 \%$ of patients. However, it restores only epicardial patency because there are small subsets of patients in whom myocardial reperfusion remains impaired despite successful restoration of patency of infarct-related artery (IRA). This is defined as no-reflow phenomenon (also known as slow flow, slow re-flow, low flow), which is largely attributed to severe microvascular obstruction (MVO) of the downstream bed [3]. Its incidence is $7 \%$ among patients undergoing rotablation, $12 \%$ with primary angioplasty, and as high as $42 \%$ for intervention of degenerative graft vessel [4]. Downstream embolisation of thrombi and plaque components during PCI mainly contribute to MVO. However, certain physical factors like spasm, edge dissection, or thrombus need to be excluded before labelling no-reflow phenomenon. It negatively affects the remodelling of infracted myocardium and may lead to hypotension, various tachy-brady arrhythmias, heart failure, and death [5-8]. Reduction of thrombus burden and its downstream migration either by mechanical means like thrombosuction or pharmacological interventions using GP Ilb/IIla antagonists, adenosine (both intracoronary and intravenous), nicorandil (intravenous and intracoronary), verapamil (intracoronary), and nitroprusside have been shown to have variable success [3]. Pharmacological agents have been shown to reduce infarct size principally through coronary vasodilatation and its anti-inflammatory effects by reducing platelet activation, calcium overload, anti-oxidant action, and neutrophil inhibition [9]. Mechanical obstruction from emboli originating from upstream lesions during $\mathrm{PCl}$ may produce antegrade resistance to arteriolar flow, which may lead to no-reflow. Forceful injection of blood through a guide catheter helps to reverse the downward spiral of no-reflow by raising the driving pressure across the capillary bed [10].

The goal of this study was to evaluate the efficacy and safety of forceful injection of blood (autologous blood transfusion - ABT) in reversing no-reflow during $\mathrm{PCl}$, because data regarding its effectiveness is not available. Primary endpoints regarding its efficacy were improvement in TIMI flow grade (achievement of TIMI 3 flow) and corrected TIMI frame count (CTFC). Safety endpoints were the absence of periprocedural complications (dissection, hypotension, air embolism) and cardiovascular endpoints (composite of stroke, acute coronary syndrome, and cardiac death) and major adverse cardio cerebrovascular events (MACCE; composite of cardiovascular (CV) events and all deaths) [11]

\section{Material and methods}

\section{Study design and participants}

This was a prospective, observational study conducted among patients who developed no-re- flow following PCI for various indications between May 2017 to December 2018 at LPS Institute of Cardiology, GSVM Medical College, Kanpur, UP, India. The indication for intervention was acute coronary syndrome which included (a) ST segment elevation myocardial infarction (STEMI) incorporating primary, pharmaco-invasive, cardiogenic shock, and rescue $\mathrm{PCl}$; (b) non-ST segment elevation myocardial infarction (NSTEMI); and (c) unstable angina (UA). Baseline demographics of patients, which included clinical (age, sex, clinical presentation and indication for intervention) and angiographic features and procedural data, were recorded. Lesions were classified as type A, B1/B2, or $C$ as per American Heart Association/American College of Cardiology (AHA/ACC) criteria [12]. The presence of collaterals (either ipsilateral or contralateral) to the infarct-related artery was graded using Rentrop grading on basal angiograms [13].

All procedures were performed after obtaining written, informed consent from all patients, and the study protocol was approved by the institutional ethical committee (GSVM Medical College EC- 81/March/2017). Major exclusion criteria for $\mathrm{PCl}$ were intolerance to antiplatelet agents (aspirin, clopidogrel, ticagrelor, prasugrel), heparin, expected major surgery within 6 months following $\mathrm{PCl}$, life expectancy $<12$ months, and pregnancy.

\section{Procedural details}

The procedures were performed through either transfemoral or transradial route following standard techniques using unfractionated heparin on a weight-based regime (70-100 $\mathrm{U} / \mathrm{kg}$ ) as an anticoagulant. Lesion modification was done except in cases of direct stenting where the lesion appeared very soft (thrombus laden) and post dilatation was performed accordingly. In the case of multi-vessel involvement in a patient with acute coronary syndrome (ACS), only the infarct-related artery was intervened during index hospitalisation. All patients were pre-treated with aspirin (325 mg) and P2Y12 inhibitors (ticagrelor, prasugrel, or clopidogrel), and dual antiplatelet (DAPT) agents were continued for at least 12 months followed by aspirin alone indefinitely. The preferred antiplatelet agent was ticagrelor, followed by prasugrel and clopidogrel depending on economy and drug availability. All patients were followed up clinically (history, electrocardiogram, and echocardiogram) at 1 week, 1 month, 6 months, and 12 months, and check angiogram was performed only if symptomatic or when they presented with acute coronary syndrome. Target vessel-related myocardial infarction (MI) was attributed to the target vessel or could not be related to another vessel on the basis of clinical presentation, laboratory data, and electrocardiogram and angiograph- 
ic findings [14]. Revascularisation was performed when the diameter of stenosis was $\geq 70 \%$ along with subjective evidence of ischaemia.

\section{Assessment of coronary flow in the catheterisation laboratory}

All angiograms were reviewed and graded independently by two interventional cardiologists to assess TIMI flow grade in accordance with the TIMI before intervention, at the onset of no-reflow, and at the end of the procedure [15]. Vasospasm, edge dissection, or thrombus were ruled out before labelling as no-reflow phenomena. Angiographic flow was assessed using corrected TIMI frame count method (cTFC) [16]. All cine angiograms were recorded at 30 frames/s with a field size that enabled visualisation of both entry of contrast into the culprit artery and its runoff from a distal landmark. It was analysed by another independent investigator in random order, who was unaware of the sequence of injections. The initial frame used for the TFC was the first frame in which contrast fully enters the artery, whereas last frame was defined as the frame when contrast first entered the branch most distal to the culprit lesion. The number of cine frames between the first and last frames was measured to determine the TFC. Intra-observer variability was assessed with two separate readings, which was \pm 5 frames. The CTFC was compared before and after autologous blood transfusion.

\section{Protocol of autologous transfusion}

In case of coronary no reflow, 100-120 ml of blood was withdrawn from the side port of a small extension tube attached to side arm of a Y-connector using multiple $10 \mathrm{ml}$ syringes over a 3- to 5-minute period with the guidewire kept in situ. All syringes were carefully de-aired and then blood was forcefully injected from the side port of Y-connector over a 3- to 4-minute period using handheld syringes. The force of injection is subjective, but it should be little more forceful than what one uses for routine contrast injection and should be enough to finish infusion of blood over 3-4 min. The amount of blood required to be infused was decided based mainly on the calibre of vessel and site of stent placement. In a medium sized vessel (> $3 \mathrm{~mm}$ ) with proximal stent, nearly $120 \mathrm{ml}$ of blood was infused while in a medium to smaller size vessel with shorter stent at mid segment of the artery, $80 \mathrm{ml}$ was infused. Electrocardiogram and vitals were closely monitored. In the case of transient hypotension, intravenous fluid was infused. TIMI flow and CTFC were finally re-assessed after $10 \mathrm{~min}$. Procedural success was defined as attainment of TIMI 3 flow. In the case of no re- sponse from ABT, standard measures like intracoronary verapamil, sodium nitroprusside, adenosine, and nicorandil were used after $15 \mathrm{~min}$.

\section{Statistical analysis}

Statistical analyses were performed using SPSS 19.0 (SPSS Inc., Chicago, IL, USA). Normally distributed data were presented as the mean \pm standard deviation (SD). Categorical data were recorded as percentages. Differences in CTFC before and after ABT were analysed using the $\chi^{2}$ test. A probability level of $p<0.05$ was considered statistically significant.

\section{Results}

\section{Patient and lesion characteristics}

During the index period 93 patients experienced no-reflow phenomenon, who were treated using ABT, among 1982 interventions performed. Baseline clinical and angiographic characteristics are shown in Table I. The patient population consisted of 66 (71\%) men and 27 (29\%) women, with a mean age of $53.4 \pm 17.9$ years. The commonest risk factor was smoking $(n=228 ; 31.9 \%)$ in the form of either cigarette or bidi $(n=29 ; 31.2 \%)$ followed diabetes $(n=24 ; 25.8 \%)$ and hypertension $(n=23 ; 24.7 \%)$. Indications for $\mathrm{PCI}$ were STEMI $(n=61 ; 65.6 \%)$, NSTEMI $(n=20 ; 21.5 \%)$, UA $(n=$ $09 ; 9.6 \%)$, and graft vessel ( $n=03 ; 3.2 \%)$. The left ventricular ejection fraction was severely impaired in 18 (19.3\%) patients, while it was relatively preserved in 55 (59.1\%) patients. The culprit vessel was left anterior descending artery in 51 (54.8\%) patients, left circumflex artery in 10 (10.8\%), right coronary artery in 29 (31.2\%), and saphenous vein grafts in $3(3.2 \%)$ patients. All grafts were degenerated (mean vein graft age: $13.3 \pm 2.1$ years). Target lesions were typically high risk (ACC/AHA class B2 or C lesions) in 78 (83.9\%) patients and mostly complex, and thrombotic (Table II).

\section{Procedural details}

The average delay from the beginning of symptoms to start of intervention was $6.2 \pm 3.5 \mathrm{~h}$ in patients with primary $\mathrm{PCI}, \mathrm{NSTEMI}$, and UA and 32.4 $\pm 12.6 \mathrm{~h}$ and $24.6 \mathrm{~h}$ in patients with rescue and pharmaco-invasive $\mathrm{PCl}$, respectively. Collaterals to the culprit artery were observed in 23 (24.7\%) patients, who were labelled as grade 3 in $10(10 \%)$, grade 2 in 11 (12\%), and grade 1 in 2 (2.7\%) patients. Thrombus aspiration was performed using a Thrombuster II (Kaneka Medical; Japan) aspiration catheter in 10 (20.4\%) patients who had acute total occlusion. Partial restoration of flow (TIMI 1/ TIMI 2) was observed in all 38 patients who had total occlusion at baseline (Figure 1). All lesions 
Santosh Kumar Sinha, Mukesh Jitendra Jha, Puneet Aggarwal, Umeshwar Pandey, Awadesh Kumar Sharma, Mahmodullah Razi, Dibbendhu Khanra, Ramesh Thakur, Vinay Krishna

Table I. Baseline, clinical, and angiographic characteristics of patients $(n=93)$

\begin{tabular}{|c|c|}
\hline Characteristics & $N(\%)$ \\
\hline Age [years] & $53.4 \pm 17.9$ \\
\hline Male & $66(71)$ \\
\hline Female & $27(29)$ \\
\hline \multicolumn{2}{|l|}{ CAD risk factors: } \\
\hline Hypertension & $23(24.7)$ \\
\hline Diabetes mellitus & $24(25.8)$ \\
\hline $\begin{array}{l}\text { Smokers (cigarette/bidi/smokeless } \\
\text { tobacco) }\end{array}$ & $29(31.2)$ \\
\hline Family history of CAD & $4(4.3)$ \\
\hline Dyslipidaemia & $20(21.5)$ \\
\hline \multicolumn{2}{|l|}{ Clinical presentation: } \\
\hline STEMI: & $61(65.6)$ \\
\hline Primary & $27(29)$ \\
\hline Pharmaco-invasive & $18(19.3)$ \\
\hline Cardiogenic shock & $5(5.3)$ \\
\hline Rescue PTCA & $11(11.8)$ \\
\hline NSTEMI & $20(21.5)$ \\
\hline UA & $9(9.6)$ \\
\hline Graft Vessel PCI & $3(3.2)$ \\
\hline \multicolumn{2}{|l|}{ LVEF: } \\
\hline$>45 \%$ & $55(59.1)$ \\
\hline $35-45 \%$ & $20(21.5)$ \\
\hline$<35 \%$ & $18(19.3)$ \\
\hline \multicolumn{2}{|l|}{ Medications: } \\
\hline Aspirin & $90(96.8)$ \\
\hline Clopidogrel & $54(58.1)$ \\
\hline Prasugrel & $24(25.8)$ \\
\hline Ticagrelor & $15(16.1)$ \\
\hline Statin & $90(96.8)$ \\
\hline$\beta$-blocker & $67(72)$ \\
\hline ACEI/ARB & $74(79.6)$ \\
\hline Ivabradine & $6(6.4)$ \\
\hline Aldosterone antagonist & $22(33.7)$ \\
\hline \multicolumn{2}{|l|}{$\begin{array}{l}\text { Angiographic severity of CAD (Target } \\
\text { vessel location): }\end{array}$} \\
\hline SVD & $49(52.7)$ \\
\hline DVD & $23(24.7)$ \\
\hline TVD & $21(22.5)$ \\
\hline \multicolumn{2}{|l|}{ Culprit lesion location: } \\
\hline $\mathrm{LAD}$ & $51(54.8)$ \\
\hline RCA & $29(31.2)$ \\
\hline $\mathrm{LCX}$ & $10(10.8)$ \\
\hline SVG & $3(3.2)$ \\
\hline
\end{tabular}

Data presented as mean \pm standard deviation or number (percentage). CAD - coronary artery disease, DM - diabetes mellitus, $P C I$ - percutaneous coronary intervention, STEMI - ST-segment elevation myocardial infarction, NSTEMI - non-ST segment elevation myocardial infarction, UA - unstable angina, LVEF - left ventricular ejection fraction, ACEI - angiotensin-converting enzyme inhibitor, $A R B$ - angiotensin-receptor blocker, SVD - single-vessel disease, $D V D$ - double-vessel disease, TVD - triple-vessel disease, $L A D$ - left anterior descending coronary artery, $L C x$ - left circumflex coronary artery, RCA - right coronary artery, SVG - saphenous vein grafts.
Table II. Procedural characteristics and outcome of patients $(n=93)$

\begin{tabular}{|c|c|}
\hline Variables & Values, $n(\%)$ \\
\hline \multicolumn{2}{|l|}{ AHA/ACC lesion class: } \\
\hline A & $2(2.1)$ \\
\hline B1 & $13(13.9)$ \\
\hline B2 & $68(73.1)$ \\
\hline C & $10(10.8)$ \\
\hline \multicolumn{2}{|l|}{ Lesion characteristics: } \\
\hline Ulcerated & $13(13.9)$ \\
\hline Thrombus & $68(73.1)$ \\
\hline Acute total occlusion & $38(40.8)$ \\
\hline Dissection & $5(5.3)$ \\
\hline Collaterals present (grade $1-3$ ) & $23(24.7)$ \\
\hline \multicolumn{2}{|l|}{ Size of vessels [mm]: } \\
\hline $2.25-2.5$ & $21(22.5)$ \\
\hline $2.5-3$ & $30(32.3)$ \\
\hline $3-3.5$ & $33(35.4)$ \\
\hline $3.5-4 \mathrm{~mm}$ & $9(9.8)$ \\
\hline \multicolumn{2}{|l|}{ TIMI flow pre procedure: } \\
\hline Grade 0 & $39(41.9)$ \\
\hline Grade 1 & $25(26.8)$ \\
\hline Grade 2 & $17(18.4)$ \\
\hline Grade 3 & $12(12.9)$ \\
\hline Median length of stent per patient [mm] & $36 \pm 12$ \\
\hline \multicolumn{2}{|l|}{ Procedural details: } \\
\hline \multicolumn{2}{|l|}{ Lesion modification: } \\
\hline Direct stenting & $10(10.8)$ \\
\hline Thrombosuction & $19(20.4)$ \\
\hline $\begin{array}{l}\text { Predilatation (semi/noncompliant } \\
\text { balloon) }\end{array}$ & $83(89.2)$ \\
\hline GP IIb/IIla antagonist & $21(22.5)$ \\
\hline Post-dilation & $80(86)$ \\
\hline $\begin{array}{l}\text { Amount of blood used for autologous } \\
\text { transfusion }[\mathrm{ml}]\end{array}$ & $98(80-130)$ \\
\hline Transient hypotension & $17(18.3)$ \\
\hline Dissection, air embolism & $0(0)$ \\
\hline CV end points: & $11(11.8)$ \\
\hline Cardiac death & $9(9.7)$ \\
\hline ACS & $2(2.1)$ \\
\hline Stroke & $0(0)$ \\
\hline MACCE & $12(12.9)$ \\
\hline TLR & $2(2.1)$ \\
\hline Stent thrombosis & $4(4.2)$ \\
\hline Corrected TIMI frame count (Pre ABT) & 53.6 \\
\hline Corrected TIMI frame count (Post ABT) & 16.9 \\
\hline \multicolumn{2}{|l|}{ TIMI flow post procedure: } \\
\hline Grade 0 & $0(0)$ \\
\hline Grade 1 & $3(3.2)$ \\
\hline Grade 2 & $13(14)$ \\
\hline Grade 3 & $77(82.8)$ \\
\hline
\end{tabular}

$C V$ end points - cardiovascular end points, MACCE - major acute cardio cerebrovascular events - composite of CV events and all deaths, TLR - target lesion revascularisation. 
were predilated except in 10 patients $(10.7 \%)$ who had a discrete ulcerated lesion where direct stenting was performed (Table II). GP IIb/IIla antagonist (Tirofiban) was used after balloon dilatation in $10(10.8 \%)$ patients, while $14(12.5 \%)$ patients received it after stent deployment following onset of no-reflow. No-reflow occurred initially after stent deployment in 66 patients, and post-stenting balloon dilatation in 37 patients, of whom 56 (60.2\%) had TIMI 1 flow while 37 (39.8\%) patients had TIMI 2 flow (Figure 1).

A mean dosage of $96 \mathrm{ml}$ of blood was forcefully injected (median: $112 \mathrm{ml}$, range: $80-130 \mathrm{ml}$ ). TIMI flow grade and CTFC after no-reflow and following $A B T$ is shown in Figures 2 and 3. A significant improvement in TIMI flow grade was observed after ABT $(p<0.01)$. TIMI flow grade improved from 1.02 to 2.52 after $A B T$ with successful restoration of TIMI 3 flow in 77 (82.8\%) patients while TIMI 2 flow was restored in 16 (17.8\%) patients. Therefore, the overall success rate of ABT was $86 \%$. No-reflow was successfully reversed in all 3 (100\%) patients with vein graft, and 74 of 90 patients (82.2\%) among native vessel intervention. Patients with the lowest TIMI flow grade after PTCA showed the most extensive improvement of coronary flow (Figure 1). Similarly, TIMI frame count decreased from $62 \pm 12$ at the time of no-reflow to $18 \pm 6$ after ABT $(p<0.001)$, with an improvement of $44 \pm 7$. In native vessels it decreased from $60 \pm 10$ to $17 \pm 5$ following ABT $(p<0.001)$, while in venous bypass grafts the TIMI frame count decreased from $64 \pm 16$ to $22 \pm 8$ after ABT $(p<0.01)$. A typical no-reflow situation of totally occluded RCA in a patient presenting with acute IWMI and its successful reversal with $A B T$ is shown in Figure 4.

\section{In-hospital and follow-up outcome}

In three patients with no-reflow, two patients had partial restoration of flow (TIMI 2) following intracoronary administration of sodium nitroprusside. They were discharged after $10.2 \pm 2.2$ days. The remaining patient with no-reflow was later mechanically ventilated because of progressive heart failure, and he later succumbed on the fifth day. However, no re-infarct or stent thrombosis were reported during index hospitalisation. On follow-up, CV events were reported in 11 (11.8\%) patients, which was primarily driven by cardiac death $(n=9 ; 9.7 \%)$. Overall death was $9.7 \%$, while death among patients who had presented with cardiogenic shock was $20 \%(n=1)$. Two patients presented with STEMI as a result of late stent thrombosis, who underwent successful revascularisation. Malignant arrhythmia $(n=4 ; 44 \%)$ and progressive pump dysfunction $(n=5 ; 56 \%)$ were reasons for all cardiac death. MACCE was reported among 12 (12.9\%) patients

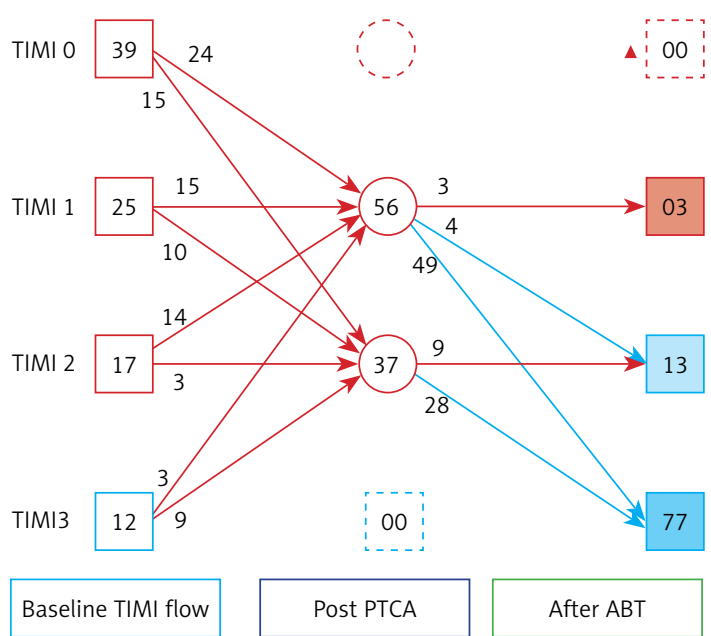

Figure 1. Changes of TIMI flow grading at baseline, after deployment of stent (all patients had TIMI flow grade $<3$ ), and following ABT, which led to its improvement to either TIMI 2 or TIMI 3 flow grade

\section{Discussion}

The present study, the first of its kind to date, demonstrated the safety and efficacy of forceful injection of blood through a guiding catheter (au-

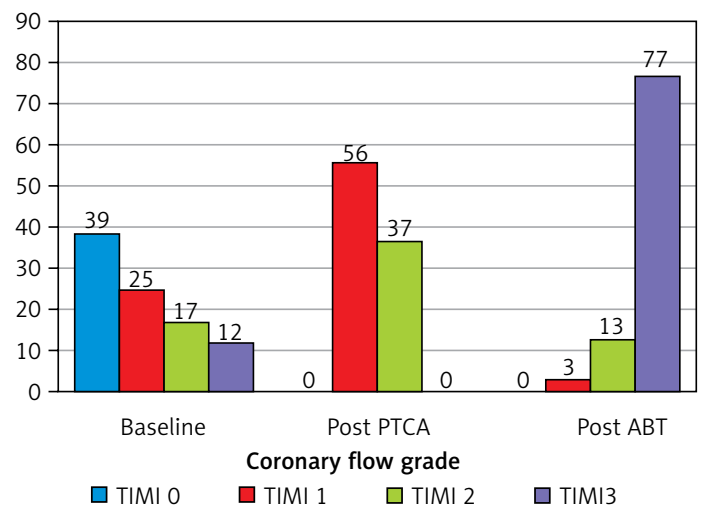

Figure 2. Changes of TIMI flow grading at baseline, after deployment of stent and after ABT. There was significant improvement in TIMI flow following ABT $(p<0.001)$ because TIMI flow was impaired in all patients prior to treatment

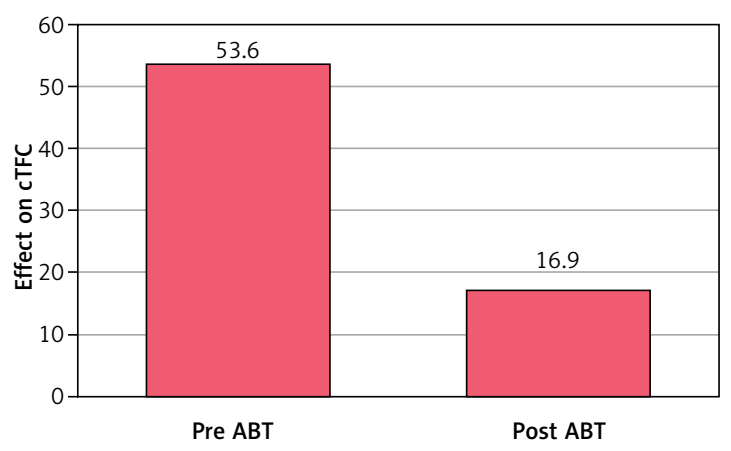

Figure 3. cTFC at the time of no-reflow and after ABT showing significant reduction in TIMI frame count $(p<0.001)$ 

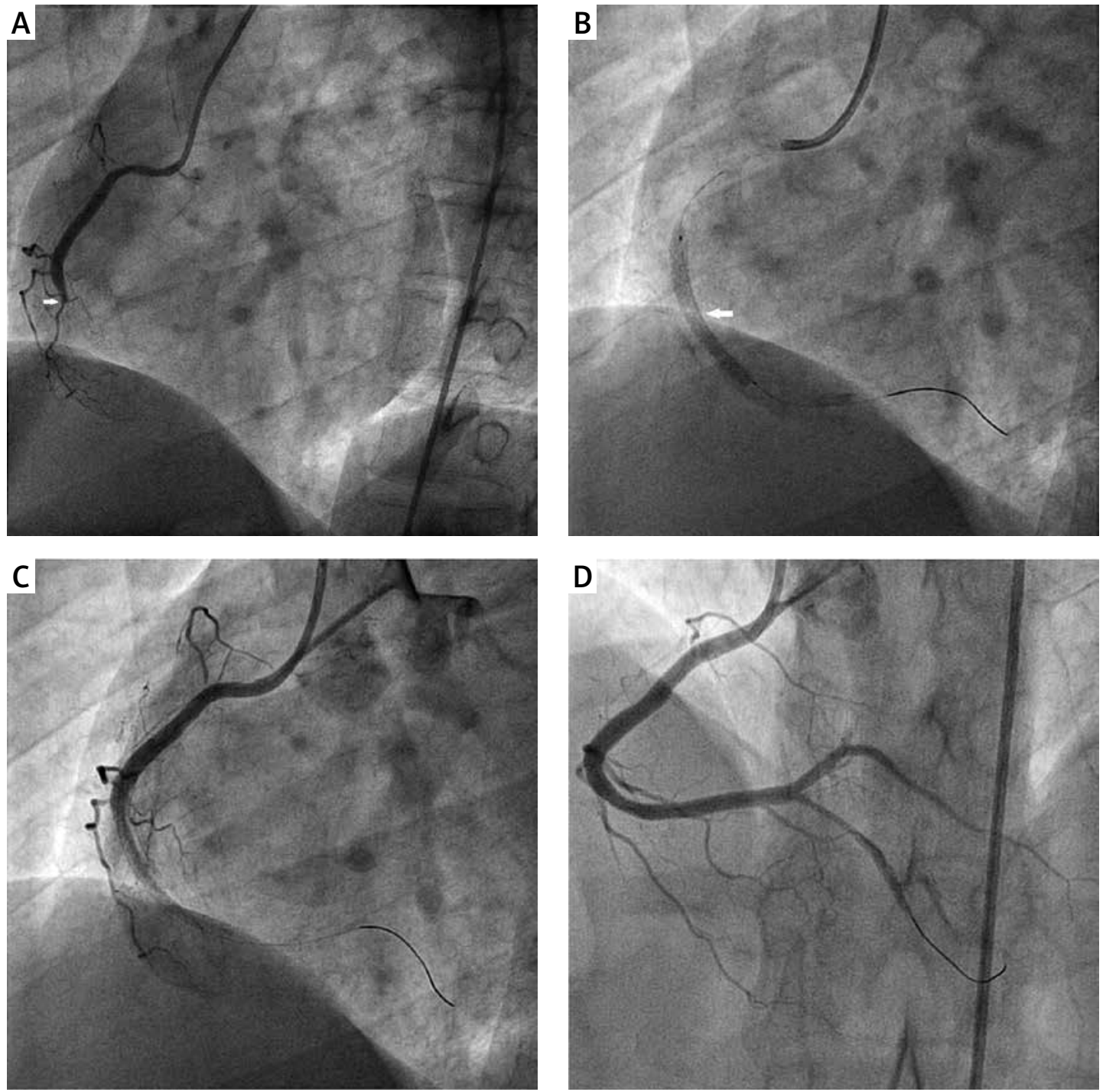

Figure 4. Left anterior oblique view showing totally occluded mid right coronary artery (A), it was stented with $3.5 \times 28 \mathrm{~mm}$ Xience Prime (Abott Vascular; USA) after deploying at $13 \mathrm{~atm}$ pressure (B), no reflow of RCA following stent deployment (C), TIMI 3 flow of RCA after forceful injection of blood (D)

tologous blood transfusion) for reversal of no-reflow during $\mathrm{PCl}$ for acute myocardial infarction. This adjunctive measure was quite safe and effective at addressing the no-reflow (overall success rate $-82.8 \%$ ), and it did not require any pharmacological intervention, thereby negating any untoward side effects, and it was a readily available option. It works not only for native vessels but also for venous grafts. There are several mechanisms of phenomenon of no-reflow, and therefore there are multiple preventive and therapeutic strategies, both pharmacological as well as mechanical, but guidelines are still lacking. This lessens the advantages of open artery and also increases the risk of future cardiovascular events, and it has been associated with worse clinical outcomes. Microvascular obstruction (size $\geq 200 \mu \mathrm{m}$ ) is the culprit and may result from clogging by atherosclerotic debris because of hardware manipulation. Underlying microvascular dysfunction, thrombus burden, and retrograde perfusion by collaterals, diabetes, and dyslipidaemia are among the exacerbating factors.

In our study, the success rate of ABT (82.8\%) was comparable to other pharmacological measures like intracoronary administration of the combination of adenosine and nitroprusside, adenosine alone, calcium channel blockers (nicardipine, diltiazem, verapamil), vasodilators (nicorandil, sodium nitroprusside), and epinephrine, the success rate of which varies from $65 \%$ to $95 \%$ [17]. The various side effects with these agents are atrio-ventricular block, ST elevation or ST depression, need of temporary pacing wire, or atropine occurring in various proportions.

Adenosine, a purine nucleoside, although effective for no-reflow, has very rapid clearance because it has a half-life of $6 \mathrm{~s}$ and therefore may require multiple administration, which is time 
consuming and costly. Also, it causes bronchospasm, chest pain, hypotension, and transient atrioventricular block $[18,19]$. ABT, in contrast to adenosine, is a one-time affair and is not associated with these side effects.

Nicorandil has the dual property of nitrate donor and ATP-sensitive potassium channel-opener, which acts on the micro-resistance vessel causing vasodilatation. It is helpful in combating no-reflow but has no action on aorto-coronary vein grafts, which might be a disadvantage, while our study has shown the effectiveness of ABT in this substrate of patients, as well [20-22].

Similarly, calcium channel blockers (nicardipine, diltiazem, verapamil) are also effective, but a major concern is the various degree of atrio ventricular block and hypotension. In this regard, ABT is superior and safer because no rhythm disturbance was noted [23-25].

Overall mortality over 12-month follow-up was concordant with Werner et al. [23], who reported it to be around $10 \%$. Our finding was also similar to results among patients who had presented with cardiogenic shock.

In our study, ABT was quite safe, although transient hypotension was reported in $18 \%$, which was reversible. The possible reason for its beneficial effect is increased driving pressure across the capillary bed, which helps clear the microvascular bed of debris, thus restoring the flow. As the density of erythrocytes and neutrophils decreases across the capillary bed, reactive oxygen species-mediated endothelial damage, inflammation, and interstitial oedema does not happen, which helps to achieve TIMI 3 flow. As the spiral of multiple factors culminate into the final phenomena of no-reflow, its treatment is multiple. As the clogging of microvascular bed is quickly cleared, the chain reaction of inflammatory cascade turning into endothelial injury and intramural haemorrhage does not occur. Or study has a complex substrate of patients, although graft vessel intervention was less, but even in this small group it was $100 \%$ successful. Therefore, we speculate that it may show its benefit in a larger cohort, as well. In our study, $22.5 \%$ of patients received GP IIb/IIla antagonists, and they benefitted from this therapy.

Although this was a prospective study of consecutive patients in whom no-reflow was observed, this was a nonrandomised study (lack of control group), had small number of patients, was observed in background of acute myocardial infarction only, and had no long-term follow-up. Moreover, acute gain in TIMI flow following forceful injection of blood confirmed its efficacy. However, one might opine about spontaneous resolution of no-reflow after prolonged observation over an extended period of time, but considering the background of $\mathrm{AMI}$ where the earliest attainment of TIMI 3 flow is the gold standard, it was not considered. Because this is the first study to be reported, it warrants further evaluation in larger randomised controlled trials.

In conclusion, the phenomenon of no-reflow is not uncommon, especially in the setting of primary percutaneous coronary intervention or intervention of bypass graft. Our study, the first ever to be reported, demonstrated the efficacy and safety of forceful injection of blood in reversing no-reflow. This modality will be newer therapeutic option in the armamentarium for the treatment of no-reflow in the catheterisation laboratory.

\section{Conflict of interest}

The authors declare no conflict of interest.

\section{References}

1. Kocayigit I, Yaylaci S, Osken A, et al. Comparison of effects of thrombolytic therapy and primary percutaneous coronary intervention in elderly patients with acute ST-segment elevation myocardial infarction on in-hospital, six-month, and one-year mortality. Arch Med Sci Atheroscler Dis 2019; 4: e82-8.

2. Chen CWR, Makkiya M, Aronow W, Spevack DM. Heightened risk of cardiac events following percutaneous coronary intervention for cocaine-associated myocardial infarction. Arch Med Sci 2020; 16: 66-70.

3. Jaffe R, Dick A, Strauss BH. Prevention and treatment of microvascular obstruction-related myocardial injury and coronary no-reflow following percutaneous coronary intervention a systematic approach. JACC Intv 2010; 3: 695-704.

4. Rezkalla SH, Kloner RA. No-reflow phenomenon. Circulation 2002; 105: 656-662.

5. Morishima I, Sone T, Okumura K, et al. Angiographic no-reflow phenomenon as a predictor of adverse longterm outcome in patients treated with percutaneous transluminal coronary angioplasty for first myocardial infarction. J Am Coll Cardiol 2000; 36: 1202-9.

6. Bolognese L, Carrabba N, Parodi G, et al. Impact of microvascular dysfunction on left ventricular remodelling and long-term clinical outcome after primary coronary angioplasty for acute myocardial infarction. Circulation 2004; 109: 1121-6.

7. Ndreppa G, Tiroch K, Keta D, et al. Predictive factors and impact of no reflow after primary percutaneous coronary intervention in patients with acute myocardial infarction. Circ Cardiovasc Interv 2010; 3: 27-33.

8. Resnic FS, Wainstein M, Lee MKY, et al. No-reflow is an independent predictor of death and myocardial infarction after percutaneous coronary intervention. Am Heart J 2003; 145: 42-6.

9. Kaul S. The "no reflow" phenomenon following acute myocardial infarction: mechanisms and treatment options. J Cardiol 2014; 64: 77-85.

10. Seth A, Quang NN, Dave V, Nguyen TN. Complications. In: Practical Handbook of Advanced Interventional Cardiology: Tips and Tricks. $4^{\text {th }}$ ed. Nguyen T, Hu D (eds.). Wiley-Blackwell, John Wiley \& Sons, New York 2013; 366-7.

11. Guzik B, Szczepanek E, Niewiara L, et al. Coronary revascularization after heart transplant - the search for prognostic factors. Arch Med Sci 2020; 16: 789-95. 
12. Guidelines for percutaneous transluminal coronary angioplasty: a report of the American College of Cardiology/ American Heart Association Task Force on Assessment of Diagnostic and Therapeutic Cardiovascular Procedures (Committee on Percutaneous Transluminal Coronary Angioplasty). J Am Coll Cardiol 1993; 22: 2033-54.

13. Rentrop KP, Cohen M, Blanke H, Phillips RA. Changes in collateral channel filling immediately after controlled coronary artery occlusion by an angioplasty balloon in human subjects. J Am Coll Cardiol 1985; 5: 587-92.

14. Lam MK, Sen H, Tandjung K, et al. Comparison of 3 biodegradable polymer and durable polymer-based drug-eluting stents in all-comers (BIO-RESORT): rationale and study design of the randomized TWENTE III multicenter trial. Am Heart J 2014; 167: 445-51.

15. Sheehan FH, Braunwald E, Canner P, et al. The effect of intravenous thrombolytic therapy on left ventricular function: a report on tissue-type plasminogen activator and streptokinase from the Thrombolysis in Myocardial Infarction (TIMI Phase I) trial. Circulation 1987; 72: 817-29.

16. Gibson CM, Cannon CP, Daley WL, et al. TIMI frame count. A quantitative method for assessing coronary artery flow. Circulation 1996; 93: 879-88.

17. Barcin C, Denktas AE, Lennon RJ, et al. Comparison of combination therapy of adenosine and nitroprusside with adenosine alone in the treatment of angiographic no-reflow phenomenon. Catheter Cardiovasc Interv 2004; 61: 484-91.

18. Assali AR, Sdringola S, Ghani M, et al. Intracoronary adenosine administered during percutaneous intervention in acute myocardial infarction and reduction in the incidence of 'no-reflow' phenomenon. Catheter Cardiovasc Interv 2000; 51: 27-31.

19. Fischell TA, Carter AJ, Foster MT, et al. Reversal of 'no-reflow' during vein graft stenting using high velocity boluses of intracoronary adenosine. Cathet Cardiovasc Diagn 1998; 45: 366-7.

20. Ito H, Taniyama Y, Iwakura K, et al. Intravenous nicorandil can preserve microvascular integrity and myocardial viability in patients with reperfused anterior wall myocardial infarction. J Am Coll Cardiol 1999; 33: 654-60.

21. Tsubokawa A, Ueda K, Sakamoto H, et al. Effect of intracoronary nicorandil administration on preventing no-reflow/slow flow phenomenon during rotational atherectomy. Circ J 2002; 66: 1119-23.

22. Kaplan BM, Benzuly KH, Kinn JW, et al. Treatment of noreflow in degenerated saphenous vein graft intervention: comparison of intracoronary verapamil and nitroglycerin. Cathet Cardiovasc Diagn 1996; 39: 113-8.

23. Werner GS, Lang K, Kuehnert $\mathrm{H}$, et al. Intracoronary verapamil for reversal of no-reflow during coronary angioplasty for acute myocardial infarction. Catheter Cardiovasc Interv 2002; 57: 444-51.

24. Weyrens FJ, Mooney J, Lesser J, et al. Intracoronary diltiazem for microvascular spasm after interventional therapy. Am J Cardiol 1995; 75: 849-50.

25. Jalinours F, Mooney JA, Mooney MR. Pretreatment with intracoronary diltiazem reduces non-Q wave myocardial infarction following directional atherectomy. J Invasive Cardiol 1997; 9: 270-3. 